\title{
BAPN-induced rodent model of aortic dissecting aneurysm and related complications
}

\author{
Tongyun Chen", Nan Jiang", Shaopeng Zhang, Qingliang Chen, Zhigang Guo \\ Department of Cardiac Surgery, Tianjin Chest Hospital, Tianjin, China \\ Contributions: (I) Conception and design: Q Chen, Z Guo; (II) Administrative support: Z Guo; (III) Provision of study materials or patients: Q Chen; \\ (IV) Collection and assembly of data: T Chen, N Jiang; (V) Data analysis and interpretation: S Zhang; (VI) Manuscript writing: All authors; (VII) \\ Final approval of manuscript: All authors. \\ \#These authors contributed equally to this work. \\ Correspondence to: Qingliang Chen, Professor; Zhigang Guo. No. 261, Taierzhuangnan Road, Jinnan District, Tianjin 300222 , China. \\ Email: leftcure@163.com; 35391185@qq.com.
}

\begin{abstract}
Background: The aim of this study was to investigate the effects of beta-aminopropionitrile (BAPN) on the arterial walls of rodents, and to analyze the gross or pathological changes of arterial and other tissues of rodents treated with BAPN at different concentrations or doses.

Methods: Eighteen SPF SD rats (4-5-week old) were divided into three groups: SD-0.2 (Group A), SD0.4 (Group B), and SD-0.6 (Group C). The groups A, B and C were given 0.2\%, 0.4\%, and 0.6\% BAPN solution, respectively, as drinking water for seven weeks. Forty SPF C57BL/6 mice (3-week old) were randomly divided into four groups: C57-0.2 (Group D), C57-0.4 (Group E), C57-0.6 (Group F) and the control group and given $0.2 \%, 0.4 \%$, or $0.6 \%$ BAPN or distilled water as drinking water, respectively, for seven weeks. All experimental animals were free to drink water. The aortas were dissected and visually examined. At the same time, hematoxylin and eosin (HE) staining was performed in aorta tissue. The vascular diameter and area of the middle membrane were measured with IPP (Image-Pro Plus 6.0).

Results: BAPN treatment significantly affected the water intake and weight gain of rats and mice. BAPN also caused thickening of the membrane in the aortas of rats and mice, and irregularity in the arrangement of elastic fibers. These pathological changes are similar to the pathological changes observed in human aneurysms. The incidence of dissecting aneurysm in C57 mice was higher than that of Sprague Dawley (SD) rats.

Conclusions: BAPN at a concentration of $0.4 \%$ was feasible to produce an animal model of dissecting aneurysm. In SD rats, the rate of pathological changes and other complications, such as intestinal rupture and scoliosis, was higher than the rates of dissecting aneurysm.
\end{abstract}

Keywords: Dissecting aneurysm; C57BL6 mice; beta-aminopropionitrile (BAPN)

Submitted Nov 06, 2020. Accepted for publication Jun 04, 2021.

doi: $10.21037 /$ jtd-21-605

View this article at: https://dx.doi.org/10.21037/jtd-21-605

\section{Introduction}

The pathogenesis of dissecting aneurysm remains unclear (1). Nowadays, most of our understanding has focused on the end-stage condition, whilst not much is known about the early stages of the condition due to the lack of applicable animal models (2). To date, the establishment of dissecting aneurysm animal models has mainly relied on mechanical means, drug induction, and gene knockdown. Furthermore, the animal models have been mainly focused on the treatment of dissecting aneurysm using surgical or interventional strategies (3). In small sized animals, the studies were mainly focused on pathogenesis, signaling pathways, and potential treatment targets and drug efficiency. BAPN, as an irreversible lysyl oxidase inhibitor, can bind with elastin or amino and alkyl groups 
competitively, which then inhibits the cross-linking between elastin and collagen, leading to development of dissection aneurysm.

At present, animal models of aortic dissection have been typically established based on surgical methods or BAPN treatment (4). Such models have contributed to investigations into the pathogenesis of aortic dissection, however, there are indeed some limitations of these models $(5,6)$. Above all, success rates were not high, which might have led to sacrifice of many animals in the experimental setting. In this study, we aimed to investigate the effects of BAPN on the arterial walls of rodents, to analyze the gross or pathological changes of the arterial walls and other tissues of rodents treated with BAPN at different concentrations or doses, and to compare these to the characteristics of human dissecting aneurysms. We present the following article in accordance with the ARRIVE reporting checklist (available at https://dx.doi.org/10.21037/jtd-21-605).

\section{Methods}

\section{Animals}

Male specific-pathogen free Sprague Dawley (SD) rats (4-5 weeks, $110 \mathrm{~g}$ ) and male C57BL6 (3 weeks) were purchased from the Institute of Health and Environmental Medicine, Academy of Military Medical Sciences. All animals underwent adaptive feeding for 3 days before further analysis. The study protocols were approved by the Ethical Committee of Tianjin Chest Hospital. Experiments were performed under a project license granted by the ethics committee of Tianjin Chest Hospital, in compliance with national or institutional guidelines for the care and use of animals.

\section{Experimental design and grouping}

Eighteen rats were randomly divided into 3 groups and were fed BAPN solution at a concentration of $0.2 \%$ (SD$0.2, \mathrm{n}=6), 0.4 \%(\mathrm{SD}-0.4, \mathrm{n}=6)$, or $0.6 \%(\mathrm{SD}-0.6, \mathrm{n}=6)$. Forty C57BL6 mice were randomly divided into 4 groups and were fed $0.2 \%$ BAPN solution (C57-0.2, $\mathrm{n}=10), 0.4 \%$ BAPN solution (C57-0.4, $\mathrm{n}=10), 0.6 \%$ BAPN solution (C57-0.6, $\mathrm{n}=10$ ), or distilled water (control, $\mathrm{n}=10$ ). All animals had free access to food and water. Daily water drinking volume was recorded, together with body weight per week.

\section{Procedures}

The animals were sacrificed after anesthesia using barbital sodium via intraperitoneal injection. The large artery was separated from the initiation of the ascending aorta to the crotch of iliac artery. Additionally, aberrant bone tissues, lung, and the ruptured intestinal wall were separated.

\section{Statistical analysis}

All normally distributed data were presented as mean \pm standard deviation. SPSS 19.0 software was used for the Student's $t$-test, and GraphPad 5.0 software was used for plotting data. Image-Pro Plus 6.0 software was utilized for the image analysis and determination of vascular parameters. $\mathrm{P}<0.05$ was considered to be statistically significant.

\section{Results}

\section{Effects of BAPN on body weight and water drinking volume}

With the increase in BAPN concentration, there was a significant increase in drug intake in both the C57 mice and SD rats, while water drinking volume and body weight showed a decreasing trend, especially in SD rats (Figure 1).

\section{Survival analysis}

With the increase in BAPN concentration, the survival of SD rats and C57 mice significantly decreased (Figure 2). The 4-week survival rates in the SD-0.2 group, SD-0.4 group, and SD-0.6 group were $83.3 \%, 50.0 \%$, and $43.3 \%$, respectively. The 7 -week survival rates in the SD-0.2 group, SD-0.4 group, and SD-0.6 group were $33.3 \%, 16.7 \%$, and $0 \%$, respectively. The 4 -week survival rates in the $\mathrm{C} 57$ 0.2 group, C57-0.4 group, and C57-0.6 group were 70.0\%, $80.0 \%$, and $50.0 \%$, respectively. The 7 -week survival rates in the C57-0.2 group, C57-0.4 group, and C57-0.6 group were $50.0 \%, 40.0 \%$, and $40.0 \%$, respectively. The survival rates in the $0.6 \%$ BAPN group showed the lowest 4-week and 7-week survival rates for both the SD rats and C57 mice.

\section{Large artery lesions}

No aneurysm was observed in the SD-0.2 group, SD0.4 group, and SD-0.6 group 4 weeks after treatment. Approximately 7 weeks after treatment, the number of 
A

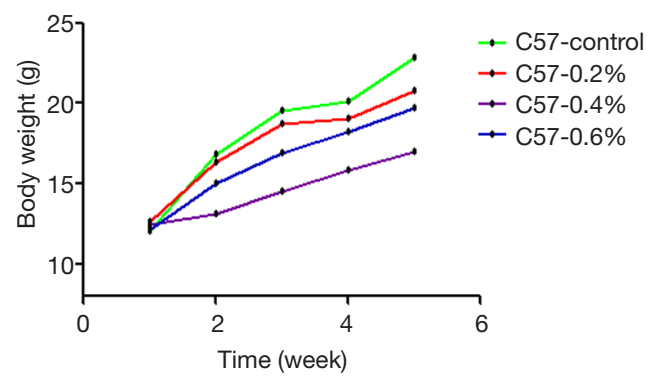

D

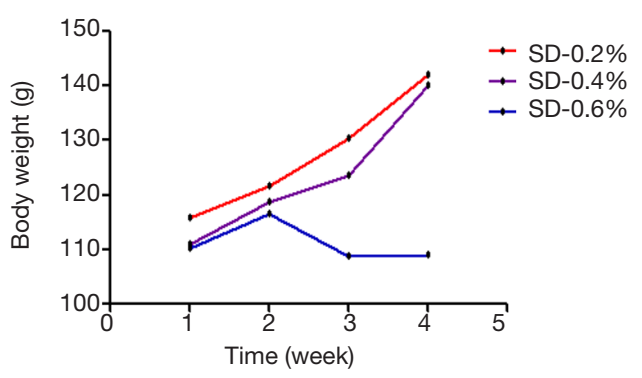

B

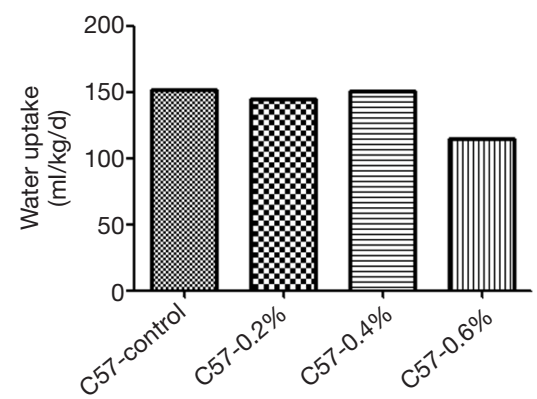

E

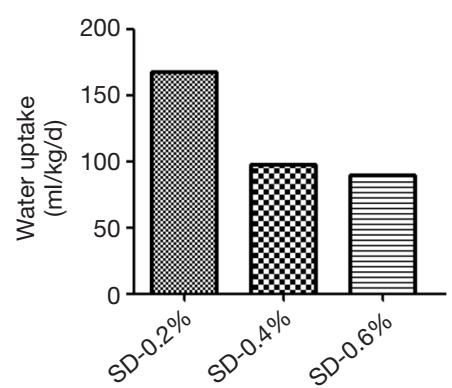

C

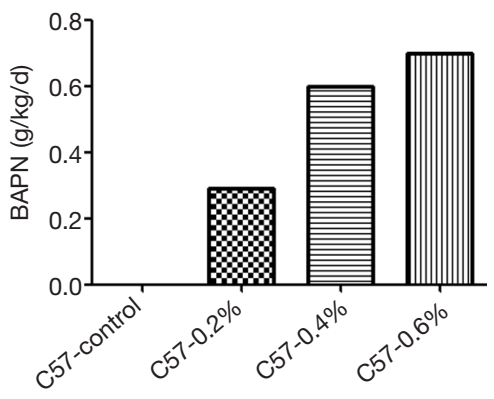

$\mathrm{F}$

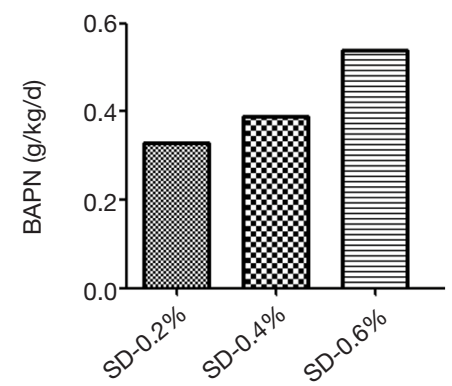

Figure 1 Changes in body weight, water uptake, and BAPN uptake in C57BL/6 mice (A-C) and Sprague Dawley (SD) rats (D-F). BAPN, beta-aminopropionitrile.

A

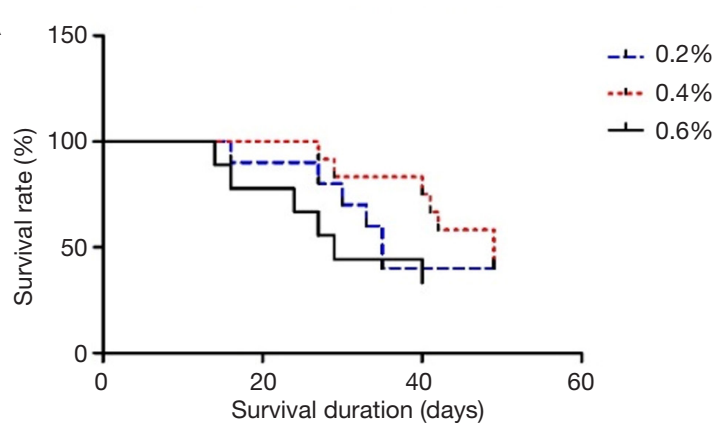

B

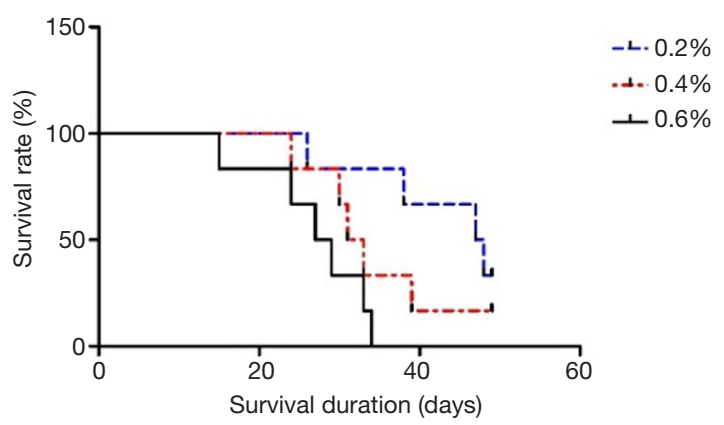

Figure 2 Survival curves of C57BL/6 mice (A) and Sprague Dawley (SD) rats (B). animals with large artery rupture or aneurysm was 1,2 , and 0 in the SD-0.2 group, SD-0.4 group, and SD-0.6 group, respectively. Among the 2 rats with aneurysms in the SD-0.4 group, 1 died from aortic rupture at week 6 . The rupture site was localized at the ascending aorta. The other rat died at week 7 from pathologically confirmed aneurysm (Figure 3). No obvious abnormalities were observed in the large arteries of other rats.

The prevalence of aneurysms at week 4 in the C57-0.2 group, C57-0.4 group, and C57-0.6 group was 10\%, 20\%, and $20 \%$, respectively. At week 7 , the prevalence of aneurysms in these groups was $50 \%, 70 \%$, and $60 \%$, respectively (Table 1). After sacrifice, 1 animal in the C57-0.2 group had pathologically confirmed aneurysm in the ascending aorta. One animal in the C57-0.4 group had pathologically confirmed aneurysm in the ascending aorta, while 2 animals had pathologically confirmed aneurysm in the descending aorta. Additionally, 2 animals in the C57-0.6 group had pathologically confirmed aneurysm in the ascending aorta (Figure 4).

The tunica media of the large artery showed thickening under the microscope, together with irregularity in the arrangement of the elastic fibers, as well as infiltration of 
A

B
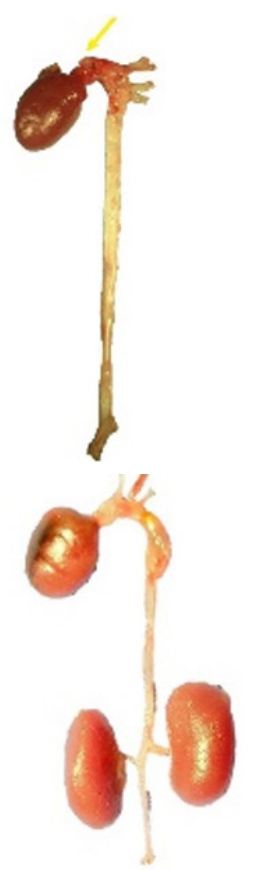
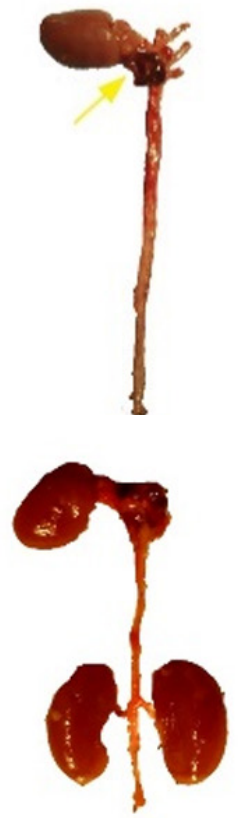
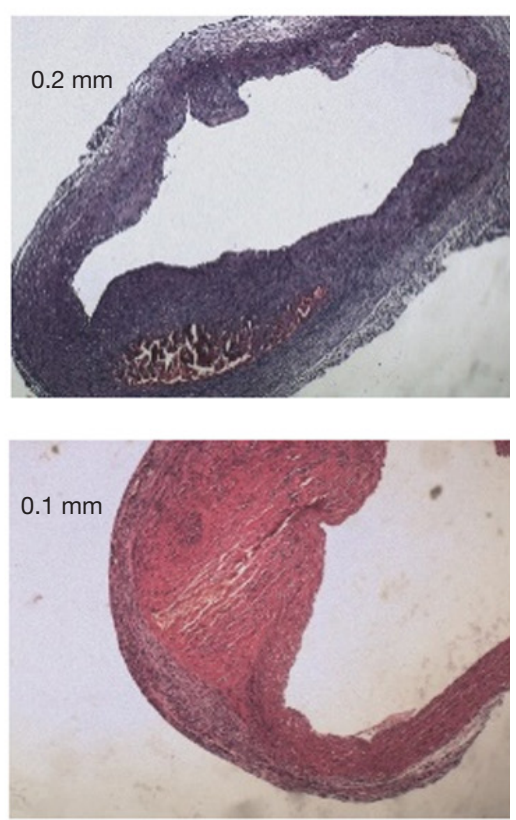

Figure 3 Macroscopic and microscopic observations of dissecting aneurysms in Sprague Dawley (SD) rats (A) and C57BL/6 mice (B). The images were observed under a magnification of $100 x$.

Table 1 Incidence of dissecting aneurysm

\begin{tabular}{lcccc}
\hline \multirow{2}{*}{ Group } & \multicolumn{3}{c}{ C57BL6 mice } & \multicolumn{2}{c}{ Sprague Dawley rats } \\
\cline { 2 - 5 } \cline { 3 - 5 } & 4 weeks & 7 weeks & 4 weeks & $16.7 \%(1 / 6)$ \\
$0.4 \%$ & $10 \%(1 / 10)$ & $50 \%(5 / 10)$ & 0 & $33.3 \%(2 / 6)$ \\
$0.6 \%$ & $20 \%(2 / 10)$ & $70 \%(7 / 10)$ & 0 & 0 \\
\hline
\end{tabular}

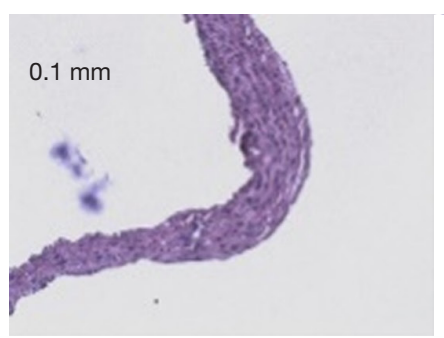

Control

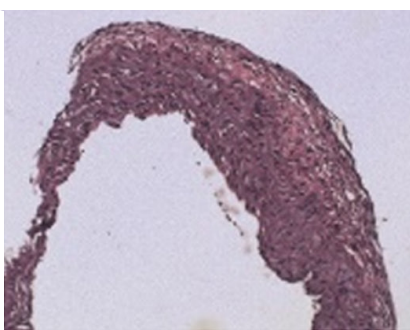

C $57-0.2 \%$

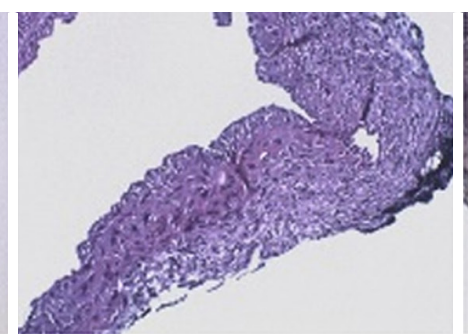

C $57-0.4 \%$

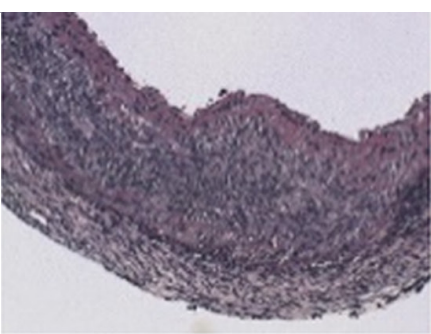

C57-0.6\%

Figure 4 Obvious thickening was noticed in the tunica media of the large artery in C57BL/6 mice. The elastic fibers were not normal in structure, and displayed rupture and disarrangement together with massive inflammatory cell infiltration. The images were observed under a magnification of $100 \times$. 

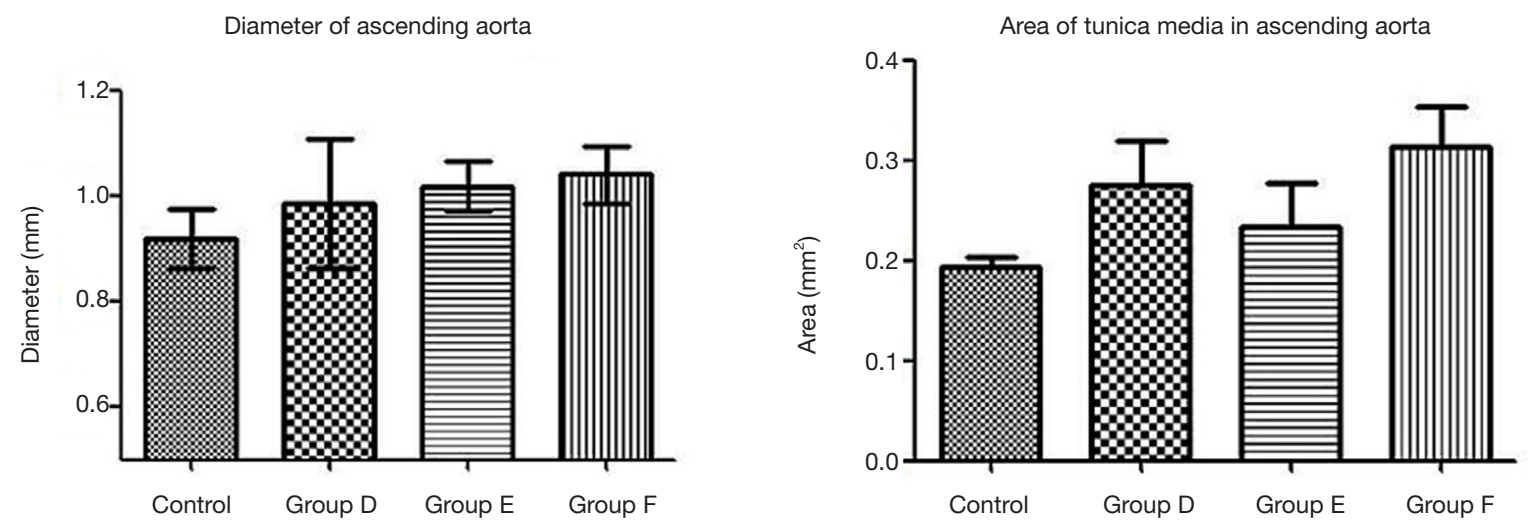

Figure 5 The diameter of the ascending aorta and tunica media area in C57BL/6 mice showed significant increases in the experimental groups compared with the control group.

Table 2 Animal complications

\begin{tabular}{|c|c|c|c|c|c|c|}
\hline Group & \multicolumn{3}{|c|}{ Sprague Dawley rats } & \multicolumn{3}{|c|}{ C57BL/6 mice } \\
\hline $0.2 \%$ & $50 \%(3 / 6)$ & $0(0 / 6)$ & $0(0 / 6)$ & $0(0 / 10)$ & $0(0 / 10)$ & $0(0 / 10)$ \\
\hline $0.4 \%$ & $33 \%(2 / 6)$ & $33 \%(2 / 6)$ & $0(0 / 6)$ & $0(0 / 10)$ & $10 \%(1 / 10)$ & $10 \%(1 / 10)$ \\
\hline $0.6 \%$ & $66 \%(4 / 6)$ & $33 \%(2 / 6)$ & $0(0 / 6)$ & $0(0 / 10)$ & $0(0 / 10)$ & $0(0 / 10)$ \\
\hline
\end{tabular}

massive red blood cells and lymphocytes. In C57 mice, the diameter of the ascending aorta and the area of the tunica media showed an increasing trend (Figure 5).

\section{Other organ changes}

In C57BL/6 mice, 1 mouse showed scoliosis in the C570.4 group. Another animal had massive pleural effusion, combined with compression and collapse of lung lobe. No other aberrant changes were noticed in the other mice.

In $\mathrm{SD}$ rats, 3 rats showed intestinal rupture after treatment with $0.2 \%$ BAPN, 2 rats showed intestinal rupture after treatment with $0.4 \% \mathrm{BAPN}$, and 4 rats showed intestinal rupture after treatment with 0.6\% BAPN (Table 2). These animals showed intestinal obstruction from the intestinal segment. Furthermore, rupture was observed in the descending colon, which resulted in severe peritonitis. Moreover, severe intestinal tympanites was observed in the jejunoileum. After dissection, scoliosis was noticed together with a tumor-like bulge in the peripheral part of the spinal column and ribs near the costovertebral joints, displaying a solid texture and white color (Figures 6 and 7).

\section{Discussion}

There are dozens of $\mathrm{AD}$ animal models, involving mice, rats, rabbits, dogs, and pigs. Modeling methods mainly include three methods: surgical or interventional instruments, drug induction, and gene knockout. Mechanical methods (e.g., surgery or intervention) including balloon dilation, doubleangle suture of internal membranes are mainly used for large animals. Drug induction is mainly used in small animal models. It has been adopted by more and more researchers as it involves simple procedure and cost-effective process. The drugs included Ang II, BAPN, mercaptoethylamine, and semicarbazide, among which Ang II and BAPN are the most widely used. Some studies involved single administration of BAPN alone, the combination of BAPN and AngII, as well as simultaneous administration of BAPN and Ang II $(4,7,8)$.

Inflammation plays a key role in the occurrence and development of aortic dissection. Macrophages presented in inflammatory infiltrates could result in aortic wall damage. The macrophages in the aneurysm wall express matrix metalloproteinase (MMP), which destroys elastic fibers and collagen fibers, and causes the aortic wall to be 

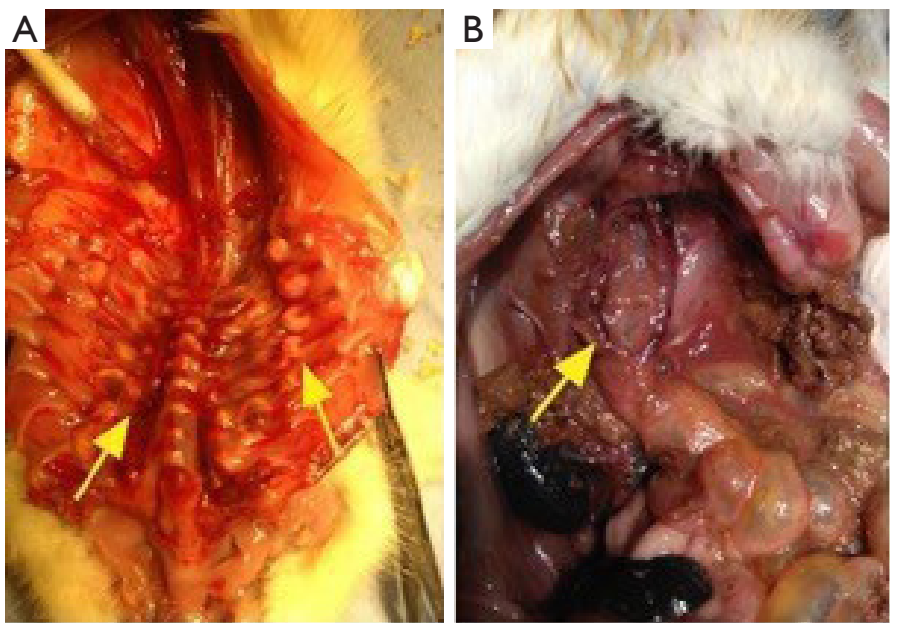

Figure 6 Scoliosis (arrow), rib tumour (arrow), and rupture position (arrow) in an animal from the Sprague Dawley (SD)-0.4 group (A) and the SD-0.6 group (B).
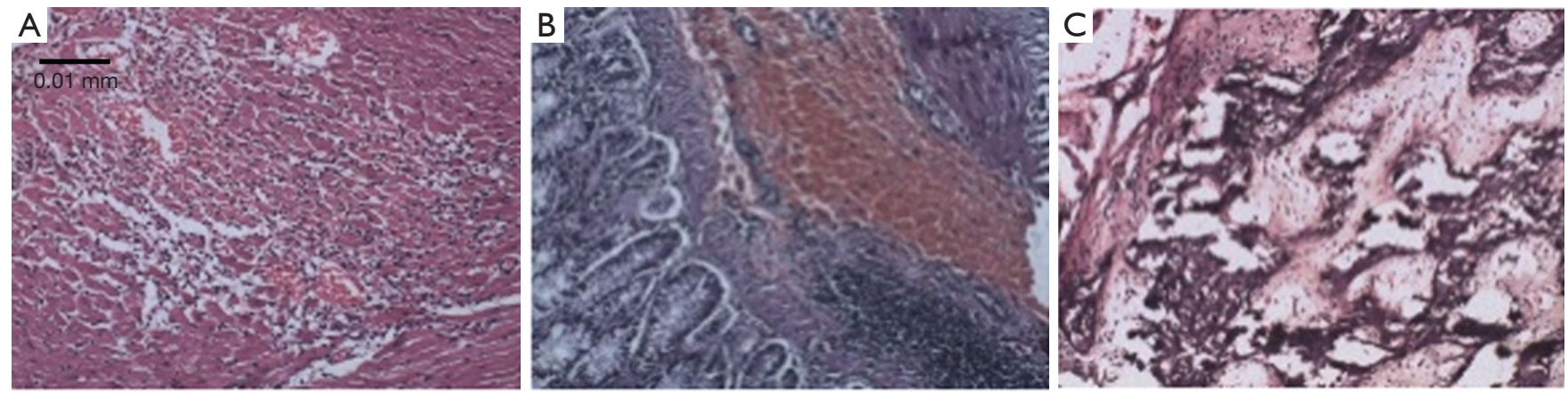

Figure 7 Pathological changes of other organs except the aorta. (A) Local myocardial cells beneath the epicardium were arranged in a loosened manner, together with infiltration of lymphocytes. (B) Separation was noticed in the tunica muscularis, together with massive red blood cells and inflammatory cell infiltration. (C) For the rib tumor, calcium salt deposition was noticed, and heterogeneous cancer cells were identified. The images were observed under a magnification of $100 \times$.

mechanically weakened. Metalloproteinases, inflammatory factors and other substances, secreted from the stimulated macrophages, can cause matrix destruction, smooth muscle cell apoptosis, all of which destroy the structure of the aortic wall $(9,10)$.

Angiotensin II can stimulate the activity of macrophages. It was reported that high level of circulating angiotensin II promoted the release of norepinephrine (NA) from sympathetic nerve endings, and then up-regulated expression of MMP-2. It is known to degrade extracellular matrix including collagen and elastin. Liu et al. (11) reported that superior cervical sympathectomy could inhibit the formation of BAPN-induced AD by inhibiting the increase of heart rate and the concentration of MMP-9 in the aortic wall.

Lysyl oxidase, with a molecular weight of 32,000 , is a monoamine oxidase with copper binding sites $(12,13)$. It can oxidate the lysine residues on collagen and elastin, which contributes to extracellular matrix (ECM) stability by promoting the covalent cross-linking of the fibrous proteins. The lysyl oxidase family consists of 5 members encoding lysyl oxidase (LOX) and lysyl oxidase-like 1-4 (LOXL1-LOXL4) $(12,14)$. They are modulated by specifically expressed genes, which results in structural and functional changes of LOX $(14,15)$. LOX has been shown to play important roles in several processes, including the stability of the ECM, inhibiting the cellular phenotype transmission induced by the Ras gene, as well as inhibiting 
the pathogenesis of cancer (16). Moreover, it has been shown to play crucial roles in maintaining the stability of ECM structure and function, organ development, trauma recovery, and cancer metastasis (17). In several malignancies including colorectal cancer, bladder cancer, esophageal cancer, and head and neck cancer, LOX family related proteins were reported to be significantly up-regulated, which was linked to promoting cancer metastasis (18).

Elastic lamina is the main component of the aortic media. Changes in its structure and composition cause changes in the mechanical and functional of the aorta. As an irreversible lysyl oxidase inhibitor, BAPN could bind with elastin or amino and alkyl groups competitively, which then inhibited the cross-linking between elastin and collagen (19). BAPN treatment can lead to thickening of the tunica media of the large artery, together with irregularity of elastin and collagen fibers, as well as necrosis. These conditions are consistent with the lesions of the large arteries in aortic dissection (AD) patients. However, the exact molecular signaling mechanisms in this process are still not clear. Studies confirmed that hypoxia, designated as a factor generally considered to be a harmful factor of disease, showed a certain protective role in the TAD model induced by BAPN (20). This mechanism of action may be related to the stimulation of HIF-1a and LOX expression by hypoxia. This may further reverse the inflammatory response and extracellular matrix (ECM) degradation.

The 3-week-old mice used in this study were at a stage of rapid growth, and BAPN might have inhibited the generation of the ECM in the large artery, which then led to the formation of aneurysms or aortic dissection. There were significant differences between the 2 species, which is considered to be closely related to their development (4). In a previous study, Ren et al. reported that the model success rate of aneurysms in FVB mice was significantly lower than that of C57 mice (4).

In this study, we selected 6-week-old C57BL/6 mice for the animal model, and no aberrant changes were observed in the large artery at week 6 after BAPN treatment. In contrast, the model success rate of 4-5-week-old SD rats was lower, with higher mortality. This may be related to the gestational age and mature development. In the C570.4 group, 1 mouse was pathologically confirmed to have scoliosis, and 1 animal showed massive pleural effusion. In SD rats, the prevalence of intestinal rupture or costal tumor was greater than the rates of aneurysm.

BAPN can trigger cardiac failure by modulating collagen (19). Furthermore, Kubota et al. reported that BAPN could lead to multiple bone malformations in chick embryos (21). Based on the molecular roles and targets of BAPN, we speculated that it might play negative roles in connective tissues rich in elastin and collagen. BAPN triggered a high success rate in establishing the animal models, leading to some functional impairment or even death. In our future studies, we will focus on the effects of mortality on the modeling process.

The prevalence of dissecting aneurysms in the C570.4 group was $70 \%$ after treatment with $0.6 \mathrm{~g} \cdot \mathrm{kg}^{-1} \cdot \mathrm{d}^{-1}$ BAPN. Using similar methods, Gao et al. established an animal model of dissecting aneurysm, which demonstrated dose-dependent effects of BAPN on the pathogenesis of dissecting aneurysm (7). In contrast, Ren et al. reported that it was not possible to establish a positive correlation between modeling success and drug intake (4). Indeed, it is still a challenge to calculate quantities after mixing BAPN with animal feed or water. Therefore, there might be significant errors in the accurate uptake of BAPN, and extensive protocols are needed to verify the stability.

There are some limitations of this study. Most of the animals used were less than 4 weeks old, and the successful establishment of the animal models was extremely low. Furthermore, the sample size was small, and the stability of the model requires multiple experiments with a large sample size. In this study, we could not perform qualitative and quantitative analysis for elastin and collagen. It will be further studied in our subsequent experiments. Statistical analysis was not possible as the data for water drinking, weight, and death were not normally distributed. Moreover, as a study involving animal models, it does not wholly represent human processes.

\section{Conclusions}

In conclusion, BAPN at a concentration of $0.4 \%$ was feasible to produce an animal model of dissecting aneurysm, with the features of low cost and convenient procedures. The prevalence of intestinal rupture and scoliosis induced lethal complications in SD rats, and these complications were significantly higher than the rates of dissecting aneurysm, which warrants further investigation.

\section{Acknowledgments}

Funding: This work was supported by Tianjin Commission of Science and Technology (No. 18ZXDBSY00160) and Tianjin Municipal Bureau of Health (No. ZC20087). 


\section{Footnote}

Reporting Checklist: The authors have completed the ARRIVE reporting checklist. Available at https://dx.doi. org/10.21037/jtd-21-605

Data Sharing Statement: Available at https://dx.doi. org/10.21037/jtd-21-605

Conflicts of Interest: All authors have completed the ICMJE uniform disclosure form (available at https://dx.doi. org/10.21037/jtd-21-605). Nan Jiang serves as an unpaid editorial board member of Journal of Thoracic Disease from Aug 2020 to Jul 2022. The other authors have no conflicts of interest to declare.

Ethical Statement: The authors are accountable for all aspects of the work in ensuring that questions related to the accuracy or integrity of any part of the work are appropriately investigated and resolved. The study protocols were approved by the Ethical Committee of Tianjin Chest Hospital. Experiments were performed under a project license granted by the ethics committee of Tianjin Chest Hospital, in compliance with national or institutional guidelines for the care and use of animals.

Open Access Statement: This is an Open Access article distributed in accordance with the Creative Commons Attribution-NonCommercial-NoDerivs 4.0 International License (CC BY-NC-ND 4.0), which permits the noncommercial replication and distribution of the article with the strict proviso that no changes or edits are made and the original work is properly cited (including links to both the formal publication through the relevant DOI and the license). See: https://creativecommons.org/licenses/by-nc-nd/4.0/.

\section{References}

1. Zhu X, Zhang X, Lu S, et al. Rare etiology for splenium of corpus callosum infarction: Anterior cerebral artery dissecting aneurysm. Neurology 2018;91:481-2.

2. Favarato D, Gutierrez PS. Case 3/2016 - 58 Year-Old Hypertensive Male with End-Stage Renal Disease, Aortic Dissection, Fever and Hemoptysis. Arq Bras Cardiol 2016;107:71-6.

3. Zhang WM, Liu Y, Li TT, et al. Sustained activation of ADP/P2ry12 signaling induces SMC senescence contributing to thoracic aortic aneurysm/dissection. J Mol
Cell Cardiol 2016;99:76-86.

4. Ren W, Liu Y, Wang X, et al. $\beta$-Aminopropionitrile monofumarate induces thoracic aortic dissection in C57BL/6 mice. Sci Rep 2016;6:28149.

5. Gawinecka J, Schönrath F, von Eckardstein A. Acute aortic dissection: pathogenesis, risk factors and diagnosis. Swiss Med Wkly 2017;147:w14489.

6. Parve S, Ziganshin BA, Elefteriades JA. Overview of the current knowledge on etiology, natural history and treatment of aortic dissection. J Cardiovasc Surg (Torino) 2017;58:238-51.

7. Gao YX, Liu YT, Zhang YY, et al. Establishment of $\beta$-aminopropionitrile-induced aortic dissection model in C57Bl/6J mice. Zhonghua Xin Xue Guan Bing Za Zhi 2018;46:137-42.

8. Fujii H, Tanigawa N, Okuda Y, Komemushi A, et al. Creation of aortic dissection model in swine. Jpn Circ J 2000;64:736-7.

9. Wang X, Zhang H, Cao L, et al. The Role of Macrophages in Aortic Dissection. Front Physiol 2020;11:54.

10. Chumachenko PV, Postnov AY, Ivanova AG, et al. Thoracic Aortic Aneurysm and Factors Affecting Aortic Dissection. J Pers Med 2020;10:153.

11. Liu H, Zheng X, Zhang L, et al. Bilateral superior cervical ganglionectomy attenuates the progression of $\beta$-aminopropionitrile-induced aortic dissection in rats. Life Sci 2018;193:200-6.

12. Kumari S, Panda TK, Pradhan T. Lysyl Oxidase: Its Diversity in Health and Diseases. Indian J Clin Biochem 2017;32:134-41.

13. Lopez KM, Greenaway FT. Identification of the copperbinding ligands of lysyl oxidase. J Neural Transm (Vienna) 2011;118:1101-9.

14. Trackman PC. Functional importance of lysyl oxidase family propeptide regions. J Cell Commun Signal 2018;12:45-53.

15. Molnar J, Fong KS, He QP, et al. Structural and functional diversity of lysyl oxidase and the LOX-like proteins. Biochim Biophys Acta 2003;1647:220-4.

16. Lucero HA, Kagan HM. Lysyl oxidase: an oxidative enzyme and effector of cell function. Cell Mol Life Sci 2006;63:2304-16.

17. Kagan HM, Li W. Lysyl oxidase: properties, specificity, and biological roles inside and outside of the cell. J Cell Biochem 2003;88:660-72.

18. Barker HE, Cox TR, Erler JT. The rationale for targeting the LOX family in cancer. Nat Rev Cancer 2012;12:540-52.

19. Fitzharris TP, Markwald RR, Dunn BE. Effects of beta- 
aminopropionitrile fumurate (BAPN) on early heart development. J Mol Cell Cardiol 1980;12:553-77.

20. Yang YY, Li LY, Jiao XL, et al. Intermittent Hypoxia Alleviates $\beta$-Aminopropionitrile Monofumarate Induced Thoracic Aortic Dissection in C57BL/6 Mice. Eur J Vasc Endovasc Surg 2020;59:1000-10.

Cite this article as: Chen T, Jiang N, Zhang S, Chen Q, Guo $\mathrm{Z}$. BAPN-induced rodent model of aortic dissecting aneurysm and related complications. J Thorac Dis 2021;13(6):3643-3651. doi: $10.21037 /$ jtd-21-605
21. Kubota S, Yuguchi M, Yamazaki Y, et al. Highly reproducible skeletal deformities induced by administration of $\beta$-aminopropionitrile to developing chick embryos. J Oral Sci 2016;58:255-63.

(English Language Editor: C. Betlazar-Maseh) 